\title{
Global Service Control for Multi-media Networks
}

\author{
Tanja de Groot ${ }^{1}$, Robert Mathonet ${ }^{2}$, David Stevenson ${ }^{3}$ \\ ${ }^{1}$ Alcatel CIT, 10 rue Latécoère, \\ 78141 Vélizy, France \\ tanja.de-groot@alcatel.fr \\ 2 Alcatel TITN Answare, 1 Rue Ampère, \\ 91747 Massy Cedex, France \\ robert.mathonet@alcatel.fn \\ ${ }^{3}$ Alcatel, 280 Botany Road, \\ Alexandria 2015, Australia \\ david.stevenson@alcatel.com.au
}

\begin{abstract}
The evolution of the market towards broadband and IP-based services is a major driving factor for network operators towards the integration of their so far separate data and telephony networks. This paper presents an approach to realize a mode of Global Service Control for telecommunication network operators that provides simplified automated management of services over a heterogeneous, multi-vendor network infrastructure. Combining existing management paradigms with new integration techniques, the Global Service Control approach makes it possible to manage carrier-class quality services over the global multi-service multi-media network.
\end{abstract}

\section{Introduction}

The trend of many incumbent operators to migrate to global multi-service networks and to provide ATM or IP-based services to business customers reflects a major change in strategy with respect to the up-to-now separate voice, data and IP networks. At the same time, operators are investing more and more in future-safe, flexible technology based operations systems (OS) that promise to provide them with

- maximum automation and control of service management solutions.

- flexibility in service definition

- speed in service activation

- security in service assurance

Defining, designing, implementing, testing and operating services on the multi-media, multi-vendor network infrastructure are as many complex tasks that impact various aspects of the operator's business, including strategy, business processes, people and technology.

For incumbent operators, the success of an evolution of his existing network to one that can support the new types of multi-media services depends on one hand on the 
ability to move their current business environment to the target convergent network porting narrowband, broadband and IP-based business environment. Investments in network evolutions will only create the expected benefits if services are successfully implemented from the perspective of the operator's customers.

This paper presents a network and service management analysis and integration approach that can serve both as the basis for the evolution of an incumbent operator's business, or for defining and realizing the business of a new operator. It is referred to as the Global Service Control solution.

The realization of a Global Service Control solution depends on the availability of an effective Integrated Network Management (INM) system. This INM system needs to provide a coherent view and control of the network resources available for services, and to provide automated processes for service activation and surveillance across the entire network.

Several Global Service Control implementations have been realized for major operators worldwide. This has made it possible to refine the approach described here to encompass multi-technology, multi-media and multi-vendor network and service management.

\section{The End-to-End Service Management Challenge}

The challenge for operators is to provide services that offer more value to their customers than those of their competitors, that may run over both public and private networks, and that provide an optimal cost/revenue ration despite the fact that these networks are in general multi-vendor, multi-technology and multi-operator in nature. This requires an operator to build a Global Service Control solution that allows him to compete efficiently in terms of:

- rapid service introduction and flexible service provisioning;

- presentation, pricing and packaging of services, which will change as market demand evolves;

- added-value services, thus reducing customer churn.

A Global Service Control solution will be the differentiating factor for the operator, making it possible to integrate a wide range of different services, and to provide a one-stop, high-speed shopping point to his customers.

The three key operational processes for achieving Global Service Control are:

- Service provisioning: Allows the operator to economically and rapidly provide a wide variety of services. This includes sophisticated pre-order processes to reliably predict what can be delivered to customers and when.

- Service assurance: Includes flexible quality of service management, allowing the operator to offer a predictable service at a reasonable cost. 
- Billing: collection and analysis of the usage data of the services, in order to charge customers for services in accordance with their actual needs and to create personalized bills.

Two conditions must be satisfied to meet these challenges: the integrated management system must provide a network-wide and customer-oriented view of the services, and the processes mentioned above must be automated as much as possible.

A phased process is required to define an operator's required business model and to achieve an actual Global Service Control solution supporting and enforcing this business model. This process must concentrate on high-speed product time-to-market, product flexibility, return on investment for the operator, and end-customer satisfaction.

\section{Divide and Conquer the Multi-technology Network}

The way to achieve a Global Service Control solution is based on a three step methodology:

\section{Step 1: Target Business Analysis}

In this step, the operator's high value products and target services (e.g. voice over packet) are clearly identified. For incumbent operators, this includes the existing products and services that first have to migrate to Global Service Control environment. Achieving this maximizes the operator's return on investment and clearly positions the operator in the customers' minds as the carrier of choice.

\section{Step 2: Definition of the Operational Process Model}

The goal of this step is to define the operator's target operational process model. In case of incumbent operators, it might include the analysis of existing operational professes. The target operational process model shall:

- provide flexible, high-speed support for the introduction of customer-oriented services;

- be easy to use (pushbutton) by the operator's employees and, optionally, by the operator's customers;

- be a smooth evolution of the existing model (incumbent operators only).

\section{Step 3: Service Evolution Realization through INM}

Step 3 requires the controlled and coordinated execution of all actions needed to realize a Global Service Control system for the selected services, as defined in step 1, and according to the target process model defined in step 2.

The goal of the Global Service Control solution is to provide a service management support according to the operators business model, that hides the complexity and diversity of the underlying network and that proposes automated processes that comply with the service operator's process model. 
Implementing the Global Service Control solution means bridging the gap between customer-oriented service management and network-oriented technology management. This requires a detailed knowledge of the actual implementation of services using the different underlying network technologies involved. A Global Service Control solution can only be realized through Integrated Network Management (INM).

\section{From Technology (TMN) to Business Management (TMF)}

Until recently, the main worry of many operators was principally the well functioning of the (mono- technology) network. Today's management systems for telecommunication networks fully support the operator's in this function. A main set of requirements for network management have been defined by a set of ITU-T recommendations.

In particular, standard M.3010 [1] defines a Telecommunication Management Network (TMN) architecture with different management layers. The Element Management Layer (EML) and Network Management Layer (NML) deal with the technology aspects of the network. They are dedicated to management of the detailed equipment aspects and network connectivity, respectively.

Furthermore, the TMN model defines the Service Management Layer (SML) and Business Management Layer (BML) to handle service activation and assurance processes and customer-related aspects and business support systems (e.g. workforce management) respectively.

The TMN model works applies well when used with mono- technology networks, such as Synchronous Digital Hierarchy (SDH) or Asynchronous Transfer Mode (ATM) networks. Technology-dedicated standardized information models specify in detail the way in which such networks should be managed at the EML level.

Only a few standards exist today that define the management of a particular technical domain at network level (NML). An example is the ATM Forum M4 Network View model that defines ATM service management on a network basis rather than on an element basis.

Furthermore, virtually no TMN standards are available today for the service (SML) and business (BML) management layers.

Finally, a major drawback of today's TMN standards is that they address technology management rather than operational activities. In particular, they do not provide service- and customer-oriented management views that are required to perform effective service provisioning, activation and surveillance. 


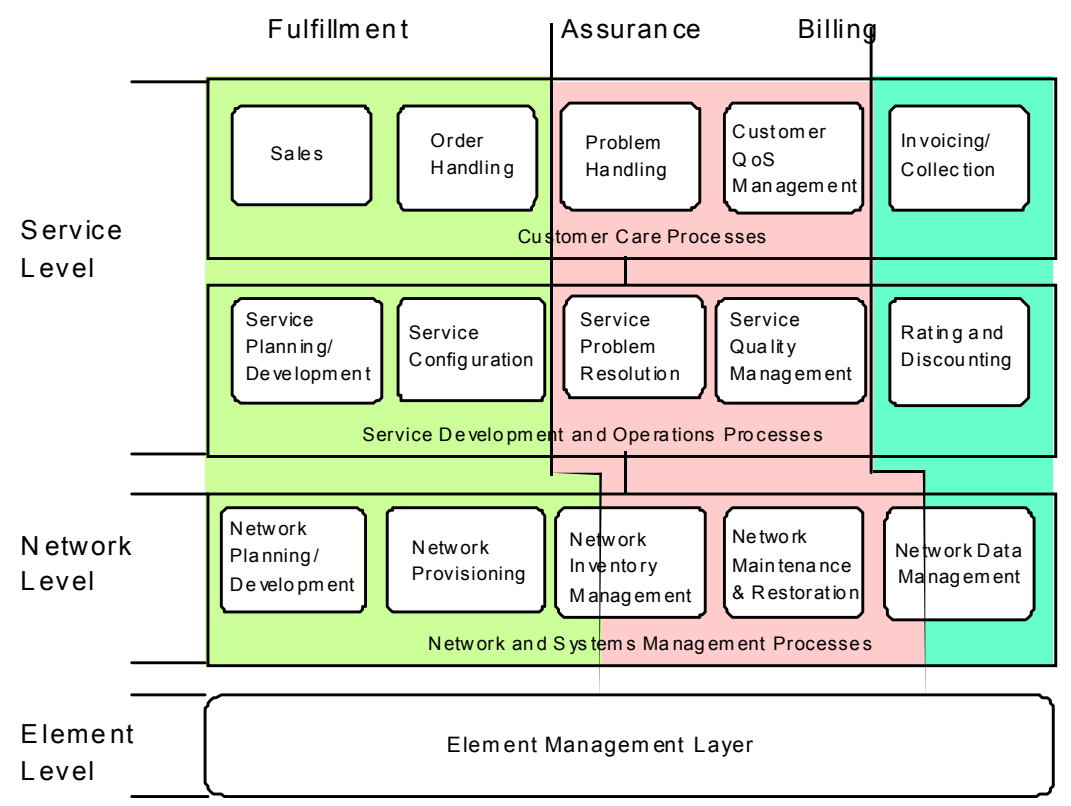

Fig. 1. TMF Operational processes on TMN architecture (TMF Operations Map)

For these reasons, the TMN model has been complemented by an operation model description, the Telecommunication Management Forum (TMF) Operations Map (TOM) ([2], see Figure 1). The TOM addresses the management problem starting from service management and operational requirements point of view.

More and more, the TOM model is used as the main reference by operators. This does not mean that the TMN functional specifications and network object-oriented models have become invalid. It mainly means that these functions now are seen as a basic given of a Network Management System, and that they serve as the basis for supporting the TOM operational processes.

\section{Service Management: From Dedicated to Cross-technology}

Apart from the fact that the accent for operator's is moving from TMN defined technology management to TMF operational process support, another shift in focus is taking place due to the arrival of convergent voice and data network architectures. The related management issue, that is not covered by today's TMN standards, is the case of managing services that may cross one or more technology boundaries.

Relations for managing the dependencies between different technologies supporting the same end-service are not defined by any standard. As an example, the impact of a fiber-optic cable break on the customers of an IP data transfer service carried over an ATM transport layer using an SDH network infrastructure is not defined anywhere. 
A combination of TMN, TMF and new models is needed to achieve a processoriented service management approach that integrates the different technology domains of the multi-vendor network into end-to-end network views and operations. Only with such a combined approach can a network management system fully support the requirements that allow an operator to achieve Global Service Control.

\section{Global Service Control Implementation}

According to the requirements listed in the previous sections on TMN technology management, TMF operational process support and cross-technology service management, Global Service Control relies on the implementation of an Integrated Network Management (INM) system, which allows to map between the objects present in the complex network infrastructure (e.g. switches, cards, ports) and the services provided on top of this infrastructure (e.g. end-to-end connections, virtual private networks (VPNs)), as shown in Figure 2.

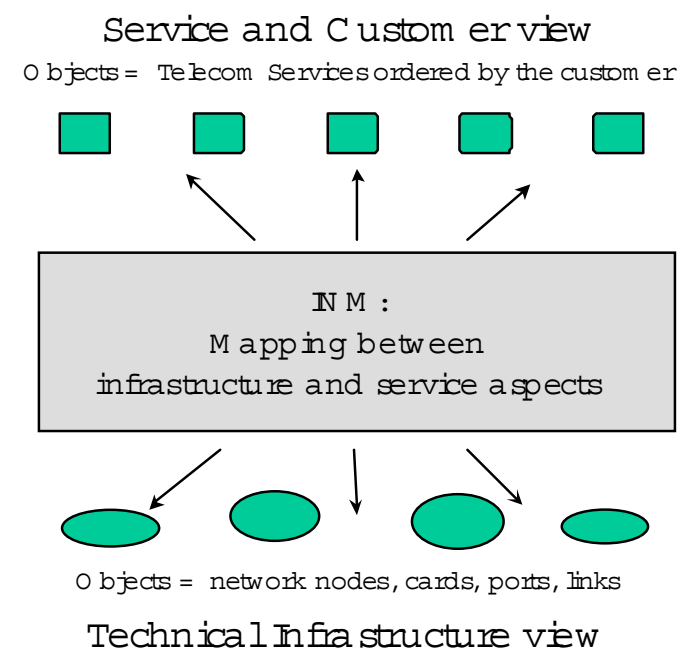

Fig. 2. The role of INM domain managers

This feature is mandatory to perform end-to-end service provisioning and activation, and to measure quality of the offered services. For example, it allows operators to determine the impact of technical faults on affected services and customers. Management processes are adapted to Service Level Agreements (SLA), helping to optimize/maximize service revenues by minimizing service outages relative to the contracted SLAs.

The key concept of such INM solutions is the Domain Manager: the tool that bridges the complexity gap between the service-oriented view and the networkoriented views. 
A Domain Manager represents an abstraction function that allows disparate technologies within the network to be managed to be handled according to business processes, rather than by technological processes.

In most cases, several levels of abstraction ( 2 or 3 ) are needed to master the complexity of the full multi-technology multi-vendor network. This is done by dividing the full network into service oriented entities, each of which is handled by a dedicated Domain Manager that makes the network to service abstraction for that part of the network.

Each Domain Manager provides the hooks down and upwards that are needed to reach the required level of service management and supporting the main operational processes for its part of the network.

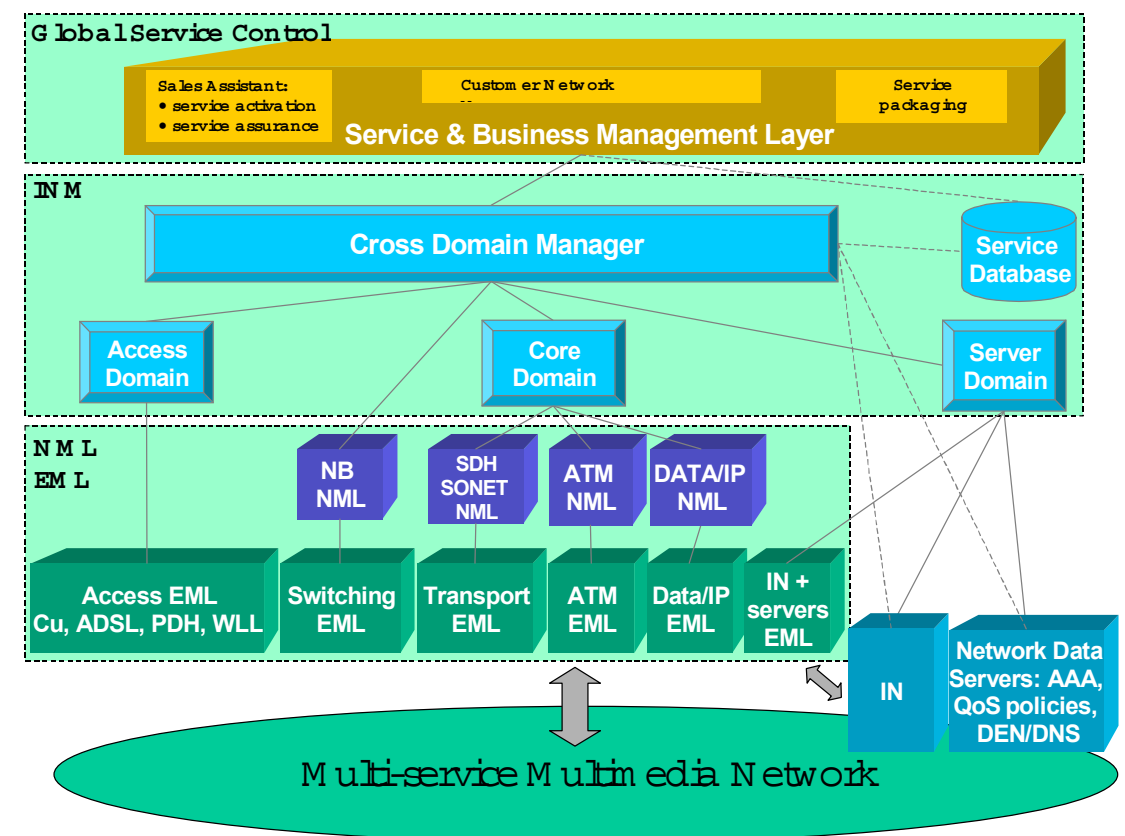

Fig. 3. Example of a Global Service Control architecture

By creating the appropriate number of Domain Managers, according to the size and complexity of the network, a divide and conquer strategy is used to master the service provisioning, assurance and billing processes across the complex network.

Thus, using the concept of Domain Manager, the shift in focus from technology management to service control is achieved. The top-level Domain Manager, also referred to as the Cross Domain Manager, At the top-level, the Cross Domain Manager interfaces with service dedicated or business management products, or with the operator's existing Operations Support System (OSS).

Figure 3 illustrates the architecture of such an INM system. This example uses three Domain Managers and a Cross Domain Manager to handle the complex 
network. Such solutions have been implemented by Alcatel for major telecommunication companies. It focuses on end-to-end service management while allowing for the use of either parallel or convergent voice and data networks.

Alcatel's experience in Domain Manager based approaches (i.e. ALMA Vision) has proven the need for the presence of carrier class technology managers (i.e. element and network technology managers, or EML/NML) as the basis on which to build the INM support (Domain and Cross-Domain Managers) needed to realize a Global Service Control solution.

Initiatives, such as the TMF Catalyst project on generic connection management, use the concept of Domain Manager as their basis.

The tendency is to use CORBA-based software platforms that enable the different modules of the solutions (Technology, Domain and Service/Business Managers) to be connected together in a flexible way. TMF CASMIM [3] is defining a serviceoriented generic network model in Interface Definition Language (IDL), which complies with TMF and TMN requirements. These interfaces are also meant to be the used to communicate with OSS applications.

The main set of functions supported by a Domain Manager are related to circuit or path management, topology management, alarm management, with a specific focus on alarms affecting circuits or paths; path performance monitoring, traffic statistics recording and SLA management.

With this kind of architecture, an operator will be able to control services over yesterday's, today's and tomorrow's network infrastructure. The approach allows an operator

- to capitalize on his existing management systems, by integrating them under a Domain Manager

- to flexibly extend or add new networks and their management systems under appropriate Domain Managers

- to make service and business management independent of the networking technology

- optionally, to migrate existing services smoothly to the convergent network architecture

\section{Conclusions}

This paper has presented the Global Service Control concept, which is crucial for operators to develop their business in a competitive environment characterized by very rapid evolution of telecom technology towards convergent networks, and by the diversity and sophistication of services, especially those proposed to corporate customers.

A methodology has been presented to achieve Global Service Control in a progressive way, starting from the operator's current situation. It has been shown that the implementation of Global Service Control requires the deployment of a carrier class INM solution to perform the mapping between technology-oriented and customer service-oriented management objects and features. 
As illustrated in the paper, the Global Service Control approach, which has been realized by Alcatel for large network operators, covers the handling

- from the service definition and customer interaction,

- through the realization of an INM system using intermediate points of control (Domain Managers) for managing service provisioning and activation, service assurance (including identification and authentication, service level agreement management, directory services, etc), and billing,

- all the way down to the technical management of the network resources.

Building an actual Global Service Control support system, requires a wide range of experience covering, amongst others, different networking techniques, service and business management and operational processes.

The methodology to reach Global Service Control applies both to today's existing services on convergent networks, as well as to the services that will be available on tomorrows multi-media networks.

The current tendency of operators to move existing services to packet based networks, and to define new services on those networks, does not change the requirements for the management of these service and the corresponding required control of the network resources and of the Quality of Service delivered by this network.

Thus, the requirements for Global Service Control remain valid in the currently foreseen network evolutions.

\section{References}

1. ITU-T: M.3010 Principles for a Telecommunications Management Network

2. Telecommunication Management Forum (TMF): TMF Operations Map

3. TMF web: http://www.tmforum.com/ for catalyst project information 